\title{
Emmanuelle LE TEXIER, Quand les exclus font de la politique. Le barrio mexicain de San Diego, Californie
}

\section{Françoise Lestage}

\section{(2) OpenEdition}

Édition électronique

URL : https://journals.openedition.org/remi/4195

DOI : $10.4000 /$ remi.4195

ISSN : $1777-5418$

Éditeur

Université de Poitiers

\section{Édition imprimée}

Date de publication : 1 octobre 2007

Pagination : 219-220

ISBN : 978-2-911627-46-6

ISSN : 0765-0752

\section{Référence électronique}

Françoise Lestage, «Emmanuelle LE TEXIER, Quand les exclus font de la politique. Le barrio mexicain de San Diego, Californie », Revue européenne des migrations internationales [En ligne], vol. $23-n^{\circ} 2$ | 2007, mis en ligne le 27 novembre 2008, consulté le 16 avril 2022. URL : http://journals.openedition.org/ remi/4195; DOI : https://doi.org/10.4000/remi.4195

Ce document a été généré automatiquement le 16 avril 2022.

(c) Université de Poitiers 


\title{
Emmanuelle LE TEXIER, Quand les exclus font de la politique. Le barrio mexicain de San Diego, Californie
}

\author{
Françoise Lestage
}

\section{RÉFÉRENCE}

Emmanuelle LE TEXIER, Quand les exclus font de la politique. Le barrio mexicain de San Diego, Californie, Paris, Les Presses de Sciences Po, 2006, 199 p., ISBN : 978-2-7246-0988-2

1 Cet ouvrage optimiste et vivifiant propose l'approche d'une jeune politologue qui veut « penser la participation des exclus loin de la culture de la pauvreté » en utilisant à la fois des méthodes d'anthropologue - la longue expérience du terrain, le regard ethnographique et le choix de la monographie - et une bibliographie de sociologue politique. Emmanuelle Le Texier souhaite briser l'image de "quartiers ethniques » où vivraient des exclus apathiques en dehors du politique, pour la remplacer par celle d'espaces sociaux actifs où les individus se mobilisent sous des formes nouvelles se développant hors de la politique conventionnelle. Ces quartiers sont appelés "barrios ", qualificatif dû à leur définition administrative tout autant qu'à la visibilité de leurs habitants mexicains ou descendants de Mexicains; un terme générique également discriminant.

2 L'auteure fonde sa démonstration sur son séjour d'un an et demi dans un faubourg pauvre de San Diego au sud de la Californie. Tout en se défendant d'offrir au lecteur une simple comparaison entre «banlieues » françaises et «barrios » étatsuniens - dont la construction historique a suivi des processus différents - elle souligne la similitude de leur position face au reste de la société nationale qui les rejette et les exclut et pose la question, bien plus large, de ce qui est politique et ne l'est pas.

3 Emmanuelle Le Texier organise son argumentation autour de deux axes principaux : les mécanismes d'institutionnalisation de la marginalité politique des habitants de ce 
«barrio » et la naissance de pratiques politiques spécifiques, notamment l'engagement des femmes. Elle montre d'une part que si les habitants du « barrio » ne participent pas au vote et n'ont pas de représentants, c'est à cause d'une sphère politique fermée aux minorités qui contribue à transformer des exclus sociaux en incompétents politiques. Elle affirme d'autre part que les femmes sont majoritairement « à l'origine de pratiques politiques au quotidien " parce qu'elles ont une vision plus optimiste que les hommes du rêve américain, mise sur le compte de la dévalorisation sociale subie par les hommes quand ils migrent aux États-Unis alors que les femmes connaissent au pire un maintien de leur statut social et au mieux, sa revalorisation.

4 Tout au long de l'ouvrage, des questions de fond sont traitées avec finesse en partant des données de terrain. Emmanuelle Le Texier soutient, par exemple, que certaines " pratiques du quotidien » comme l'engagement associatif relèvent du politique et elle refuse d'opposer un domaine politique public à une sphère non-politique privée, division qui constitue, à ses yeux, un "modèle masculin du pouvoir " alors que la distinction entre public et privé est déterminée « par l'histoire et le contexte » (p. 115). Elle s'interroge sur la représentation ethnique prônée aux États-Unis : qui est légitime? pour représenter qui ? ce système avantage-t-il ou non les exclus? Elle analyse avec finesse le jeu complexe des identifications individuelles (mexicano, raza, neo-latino, chicano, latino, mexican-american, hispanic) et leur rapport avec des prises de position politiques, toujours dans un va-et-vient entre les données de terrain et des lectures solides et bien choisies.

De la thèse de doctorat en sciences politiques dont il est issu, ce livre a conservé des mises au point synthétiques des débats relatifs aux questions sociologiques ou politiques abordées, comme celles des théories sur la création d'enclaves ethniques ou sur la participation des exclus. Ces mises au point ont le mérite de traduire en termes clairs le jargon de la sociologie politique et présentent une grande utilité pédagogique pour les lecteurs néophytes et les étudiants; tout comme les références à une kyrielle d'auteurs états-uniens peu lus en France ou carrément inconnus qu'Emmanuelle Le Texier fait ainsi connaître.

6 En s'attachant exclusivement à l'inscription politique locale des habitants d'un quartier «mexicain » de San Diego, l'auteure propose un nouvel éclairage sur l'insertion sociale et politique des migrants et de leurs descendants et prend ses distances avec les problématiques habituelles bi ou transnationales. C'est là tout l'intérêt du livre. Mais ce parti pris pose aussi question: on a le sentiment que ce «barrio» est un isolat géographique et politique et que ses résidents n'ont pas de passé. On aimerait en savoir plus sur les liens sociaux et politiques que les habitants de ce quartier, tout proche de la frontière mexicaine, maintiennent avec le Mexique et les autres associations du même type en Californie du Sud ainsi que sur leurs trajectoires de militant(e)s. Peut-être la matière d'un prochain livre? 


\section{AUTEURS}

\section{FRANÇOISE LESTAGE}

Université de Paris 7, Unité de Recherche Migration et Sociétés, francoise.lestage@univ-parisdiderot.fr 